\title{
Integrated Nanogenerators in Biofluid
}

School of Materials Science and Engineering, Georgia Institute of Technology, Atlanta, Georgia 30332-0245

Received May 28, 2007

\begin{abstract}
We have demonstrated a prototype $\mathrm{ZnO}$ nanowire based nanogenerator (NG) that can effectively generate electricity inside biofluid when stimulated by ultrasonic waves. The potential of increasing output current and voltage was illustrated by connecting multiple NGs in parallel and serial, respectively, clearly demonstrating the possibility of raising output power by three-dimensional integration and architecture. The output current was increased by 20-30 times and reached as high as $35 \mathrm{nA}$ when a $2 \mathrm{~mm}^{2}$ size NG was placed at a region where the ultrasonic waves were focused. This work unambiguously shows the feasibility of NGs for power conversion inside biofluid. It sets a solid foundation for self-powering implantable and wireless nanodevices and nanosystems in biofluid and any other type of liquid.
\end{abstract}

Self-powered nanosystems are of great importance for realtime and implantable biosensing, environmental monitoring, electromechanical systems, and personal electronics. ${ }^{1-3}$ There are two ways for achieving self-powered nanosystems. One is to use a battery, but the main challenge is to manufacture long lifetime, small size, and possibly light weight batteries. For biomedical applications, one has to consider the toxicity of the materials that compose batteries. The other approach is to harvest energy from the environment by converting mechanical, chemical, or thermal energy into electricity. ${ }^{4}$ Approaches have been developed for generating energy using thermoelectrics, ${ }^{5}$ piezoelectrics, ${ }^{6}$ bacterial organisms, ${ }^{7}$ and solar cells. ${ }^{8}$ For in vivo medical applications, the requirement is that the energy converter harvests energy from biological systems and works in biofluid for lifetime operation.

We have developed a direct-current nanogenerator that is driven by ultrasonic waves. ${ }^{9}$ The basic principle is to use piezoelectric and semiconducting coupled nanowires (NWs), such as $\mathrm{ZnO}$, to convert mechanical energy into electricity. ${ }^{10,11}$ This nanogenerator (NG) has the potential of directly convert hydraulic energy in the human body, such as flow of body fluid, blood flow, heart beat, and contraction of blood vessels, into electric energy. Although a prototype of the NG has been demonstrated by indirect driving using ultrasonic waves through a metal frame suspended in air, ${ }^{9}$ it is essential to develop nanogenerators that work in water or biofluid.

In this paper, we present an improved design of NG that is able to generate electricity in biocompatible fluid as driven by ultrasonic waves. The output current of the NG has been improved by a factor of 30. By integrating several NGs, we have demonstrated the potential of improving output voltage

* To whom correspondence should be addressed. E-mail: zlwang@ gatech.edu. and current. This research sets the platform toward threedimensional architectured NGs as practical power sources.

The NG was modified from our original model composed by vertical aligned $\mathrm{ZnO} \mathrm{NWs}$ and a Pt-coated zigzag top electrode, as described in detail elsewhere. ${ }^{9}$ The NG core was completely packaged by a polymer to prevent the infiltration of biofluid into the NG. The polymer also has certain flexibility to remain free of relative movement between $\mathrm{ZnO}$ NWs and the top of the electrode. As shown in Figure 1a, the NG was placed inside a container filled with $0.9 \% \mathrm{NaCl}$ solution, which is a typical biocompatible solution. The substrate and the top electrode were connected by waterproof extension cord to the outside of the container and marked as the positive and negative electrode, respectively. The definition of the two electrodes is according to the flow of current in the external circuit, which is always from the bottom substrate to the top zigzag electrode owing to the rectifying effect of the Schottky barrier between $\mathrm{ZnO}$ and $\mathrm{Pt}$ in the core of the NG. ${ }^{10}$ An ultrasonic stimulation was applied through the bottom of the container with a frequency of $\sim 41 \mathrm{kHz}$. The ultrasonic wave transported through the fluid and triggered the vibration of the electrode and NWs to generate electricity. For the following studies, the size of the NG was about $2 \mathrm{~mm}^{2}$, on which there are more than one million NWs.

The performance of the NGs was characterized by measuring short circuit current $I_{\mathrm{sc}}$ and open circuit voltage $V_{\mathrm{oc}}$. The output of the NGs is in the nanoampere and millivolt level. Measuring electric signal at such a small scale is always challenging because of system and/or environmental interference, such as system capacitance, thermal/instability drifting, and the bias of the amplifier. Caution must be taken at each step to ensure the signal was generated by the NG instead of the measurement circuits. We found that a "double- 

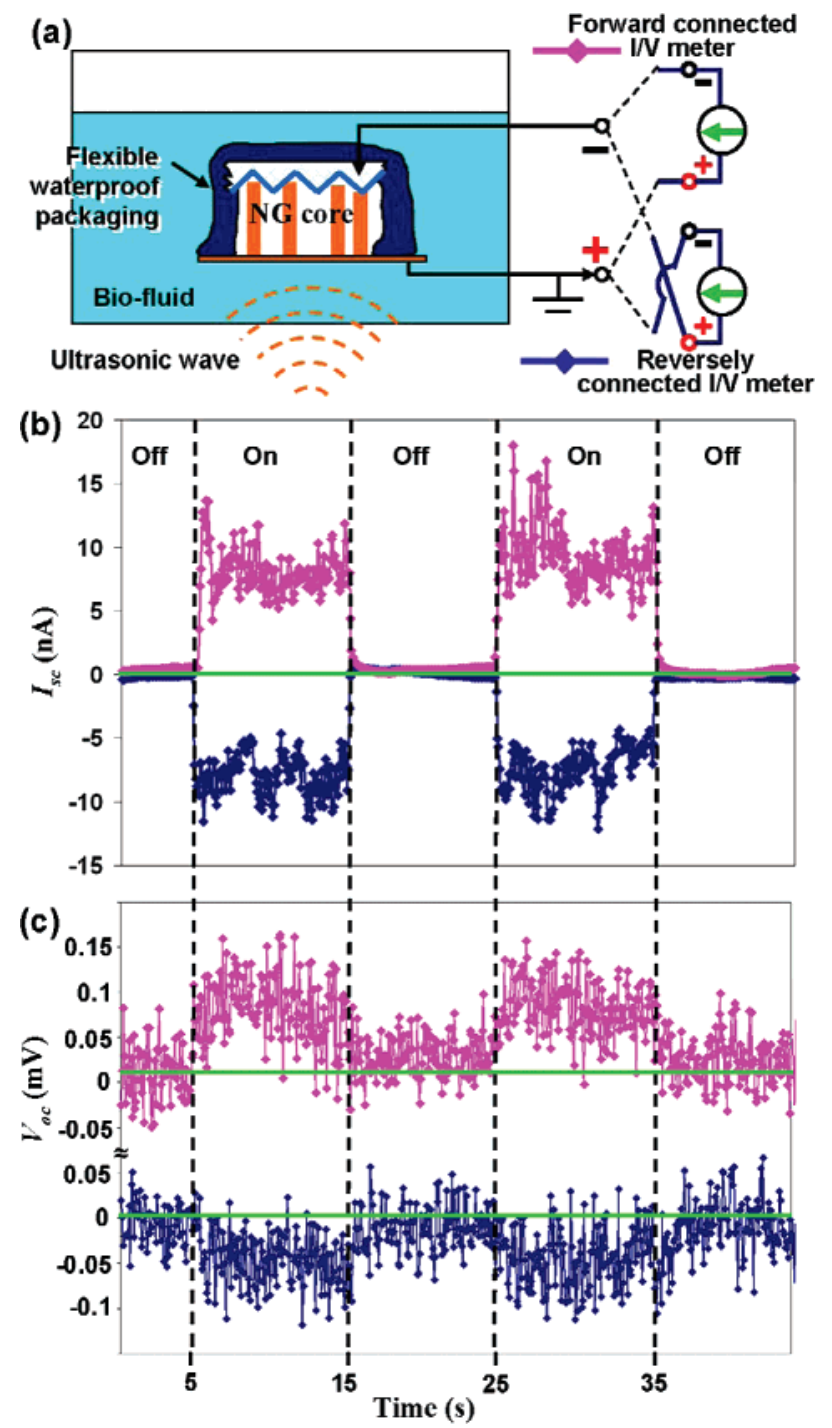

Figure 1. (a) Schematic of a NG that operates in biofluid and the two types of connections used to characterize the performance of the NG. The pink and blue curves represent signals from forward connected current/voltage $(I / V)$ meter and reversely connected $I / V$ meter, respectively. (b, c) The short circuit current and open circuit voltage measured by the two types of connections when the ultrasonic wave was turned on and off.

side" testing was very effective for ruling out artifacts. As illustrated in Figure 1a, during the measurements, the current/ voltage meter was forward connected (positive and negative probes were connected to the positive and negative electrodes, respectively) and reversely connected (positive and negative probes connected to negative and positive electrodes, respectively) to the two electrodes of the NG. The corresponding $I_{\mathrm{sc}}$ and $V_{\mathrm{oc}}$ signals are shown in parts b and c of Figure 1, respectively. After an ultrasonic wave was switched on, current output peak reached as high as $17 \mathrm{nA}$, with an average output of $\sim 9 \mathrm{nA}$ and a "noise/ instability" within $\pm 4 \mathrm{nA}$ (pink line in Figure 1b). When the current meter was reversely connected, current signal with the same amplitude was received but reversed in sign (blue line in Figure 1b). This measurement clearly eliminated the effects from measurement system error and confirmed that the current was generated by the NG.
The corresponding voltage output is rather low and noisy compared to the current signal. As shown in Figure 1c, a typical voltage level is $\sim 0.1 \mathrm{mV}$, and a jump of the signal can still be clearly distinguished from the baseline. The voltage exhibited the same sign as the corresponding current signal, and its sign was reversed while the meter connection was switched. Previous atomic force microscopy (AFM) deflection of a single NW generated a voltage output in the range of $5-10 \mathrm{mV},{ }^{11}$ which is much larger than that of the NG as driven by ultrasonic waves. The low voltage could possibly result from two reasons. First, the bending angle of a NW as deflected by an AFM tip with direct contact was much larger than that produced by an indirect ultrasonic wave. From theoretical calculation, the piezoelectric voltage produced by a NW is proportional to its lateral deflection. Therefore, AFM can generate much higher voltage between the ZnO NW and its tip. Second, although the parallel packaged NW array as shown in Figure 1a gives, in principle, a similar voltage output as a single NW used in AFM deflection, a large difference in capacitance between the two cases may result in much reduced voltage output. The capacitance of the NG shown in Figure 1a was measured to be $\sim 18-22 \mathrm{pf}$ in the frequency range of $75 \mathrm{kHz}$ to $1 \mathrm{MHz}$, while the capacitance of a single NW and the AFM system used in our previous study was $\sim 1.2$ pf. ${ }^{10}$ More than $97 \%$ of the NWs in the NG were not active for producing current but did serve as capacitors for storing changes created by the active NWs $(<3 \%){ }^{9}$ The large capacitance effectively reduces the output voltage. Therefore, the output voltage could be reduced by $\sim 20$ times for the NG. This is probably why the output voltage of the $\mathrm{NG}$ was $\sim 0.5 \mathrm{mV}$ or even smaller.

For technological applications, raising the voltage and current outputs of the NG are essential for raising the output power. If we take each NG as a battery, the most straightforward way to increase the current/voltage is to put them in parallel/serial. This has been demonstrated experimentally. As shown in parts a and b of Figure 2, two NGs I and II have been tested under the same experimental conditions. NG I exhibited an average $I_{\mathrm{sc}}$ of $\sim 0.7 \mathrm{nA}$, and NG II showed a lower noisy signal of $1 \mathrm{nA}$. These two NGs were then connected in parallel (inset of Figure 2c) and tested under the same condition again. The resultant output current reached an average of $\sim 1.8 \mathrm{nA}$, which is the sum of the two individual outputs (Figure 2c). This concept was further proved by antiparallel connecting NG I and NG II (inset of Figure 2d). Since the magnitudes of the $I_{\mathrm{sc}}$ of the two NGs were very close, the total current was mostly cancelled out by the "head-to-tail" connection in parallel, and the received signal was around the baseline (Figure 2d), just as we expected.

If more NWs were located in the activate position when packaging the top zigzag electrode, the output current can be improved by several times. As shown in Figure 2e, with a better assembly, NG III exhibited an average $I_{\mathrm{sc}}$ of $\sim 4$ nA. Once NG III was added to the parallel connection with NG I and NG II (inset of Figure 2f), their total output current was the sum of the three NGs $(5.9 \mathrm{nA} \approx 0.7 \mathrm{nA}+1 \mathrm{nA}+$ 


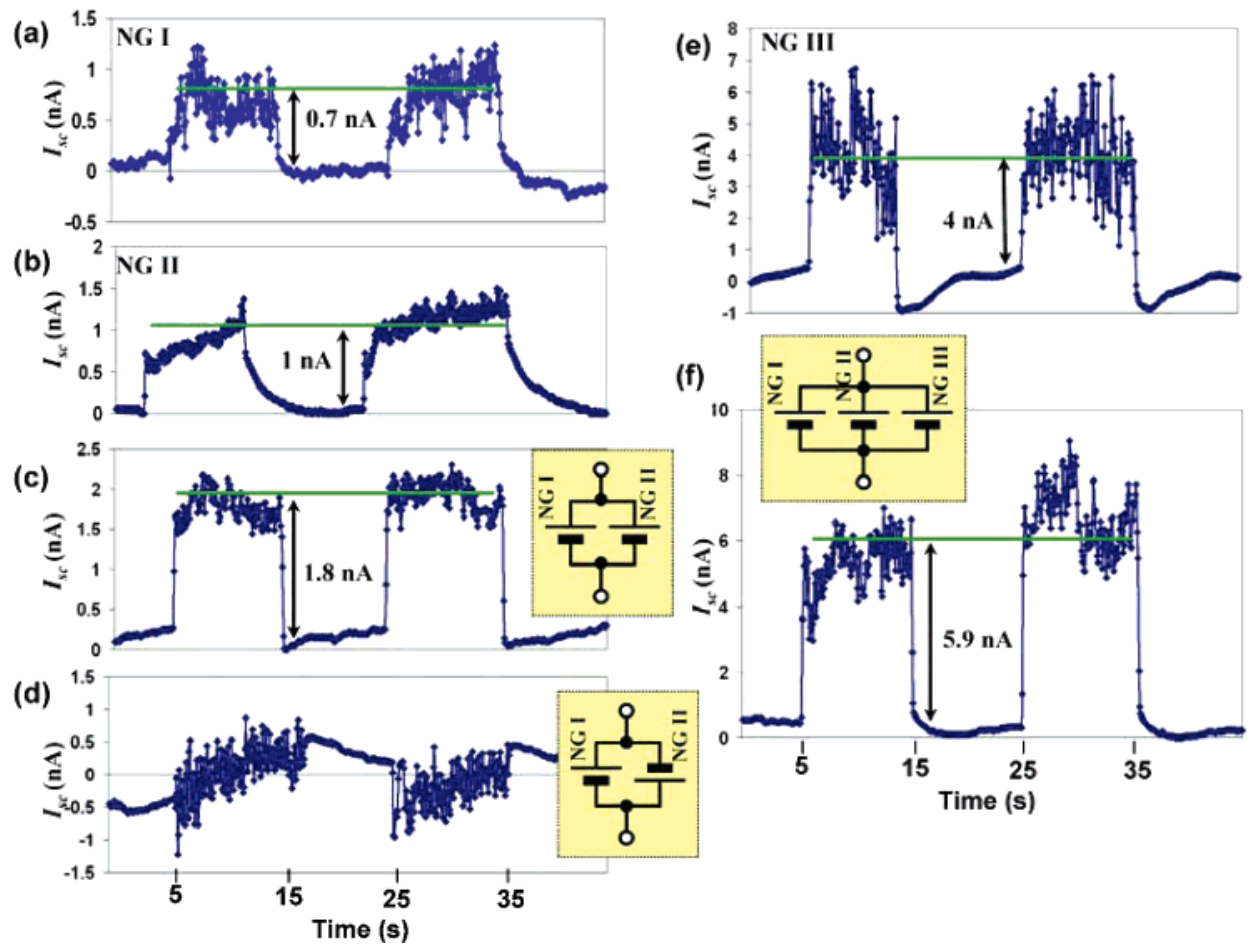

Figure 2. Short circuit current measured from an integrated NGs system. (a, b) Current signal measured from two individual NGs, I and II. (c, d) Current signal measured from parallel and antiparallel connected NG I and NG II, respectively; the connection configurations are schematically shown in the insets. (e) Current signal measured from NG III with a better performance. (f) Current signal measured from parallel connected NG I, II, and III; inset shows the connection configuration.

$4 \mathrm{nA}$ ), as shown in Figure 2f. This series of experiments demonstrated that the short circuit current can be effectively improved by parallel connecting multiple NGs. This sets the platform for developing three-dimensionally stacked NGs.

Although the voltage signals were much noisy than current, as explained before, ${ }^{9}$ the increasing trend can still be clearly distinguished when NGs were connected in serial. As shown in Figure $3 \mathrm{a}$, a single NG gave a very noisy voltage output of only $\sim 0.04 \mathrm{mV}$ (NG I). When two similar NGs (I and II) were connected in serial, their output voltage was almost doubled to an average value of $0.07 \mathrm{mV}$ (Figure 3b). Once the NG III was added to the serial, the resultant voltage output clearly jumped above the baseline and reached an average of $\sim 0.18 \mathrm{mV}$. Therefore, similar to the current output, the open circuit voltage can be effectively improved by connecting multiple NGs in serial. Although the individual voltage output might be low, microfabrication techniques can be used to integrate hundreds of NGs onto one chip or stack them vertically for receiving high output voltage.

The local intensity of the ultrasonic wave was important for improving the output of the NG. As schematically shown in Figure 4a, the biofluid was placed into a glass container with a diameter of $11 \mathrm{~cm}$ and a height of $9.5 \mathrm{~cm}$. The ultrasonic wave source was placed at the center beneath the container. Once the ultrasonic wave was excited inside the container, it was reflected by the container's wall and the water surface. As a result, the wave intensity was enhanced in a certain region inside the fluid. A NG was placed inside the fluid and held by a clamp that can be freely moved in any direction to trace the enhanced ultrasonic wave, while the output current was continuously monitored. First, the NG was placed at the center region above the water surface and the ultrasonic wave was kept on. The corresponding current signal is shown in Figure 4b. Then the NG was slowly moved into the fluid along the $z$ direction (depth direction) and a jump in current for $\sim 1 \mathrm{nA}$ was immediately detected once the NG touched water surface. When the NG reached $\sim 3.3 \mathrm{~cm}$ below the water surface, the current quickly jumped to $\sim 20 \mathrm{nA}$. The output can be kept at such a high level as long as the NG stayed at this depth. After $15 \mathrm{~s}$ of steady high output, the NG was moved further down and the current dropped back to the 1-2 nA level again. When the bottom of the container was reached, the NG was pulled upward to the water surface. The same $20-25 \mathrm{nA}$ high current output was observed again once the NG reached $3.3 \mathrm{~cm}$ depth. The current signal dropped back to its baseline after the NG was pulled out of water.

Then, the NG was kept $3.3 \mathrm{~cm}$ below water surface but moving along the $x$ and $y$ directions. The output current is shown in Figure 4c. Starting at the central region, when the ultrasonic wave was turned on, the current quickly jumped to $25 \mathrm{nA}$ and soon reached the highest peak of $35 \mathrm{nA}$. Then the NG was slowly moved away from the central region while its vertical position was kept unchanged. The current quickly dropped to $\sim 1 \mathrm{nA}$ when the NG was $\sim 2-3 \mathrm{~cm}$ away from the center point. The NG was continuously moved from left to right and from front to back. Only when it passed through the central region of the container, the high current output of $\sim 20 \mathrm{nA}$ was detected. These two experiments illustrated how the intensity of ultrasonic wave dictated the NG's output. At the center and $3.3 \mathrm{~cm}$ inside the fluid, the short circuit current was increased by $20-30$ times. This is 

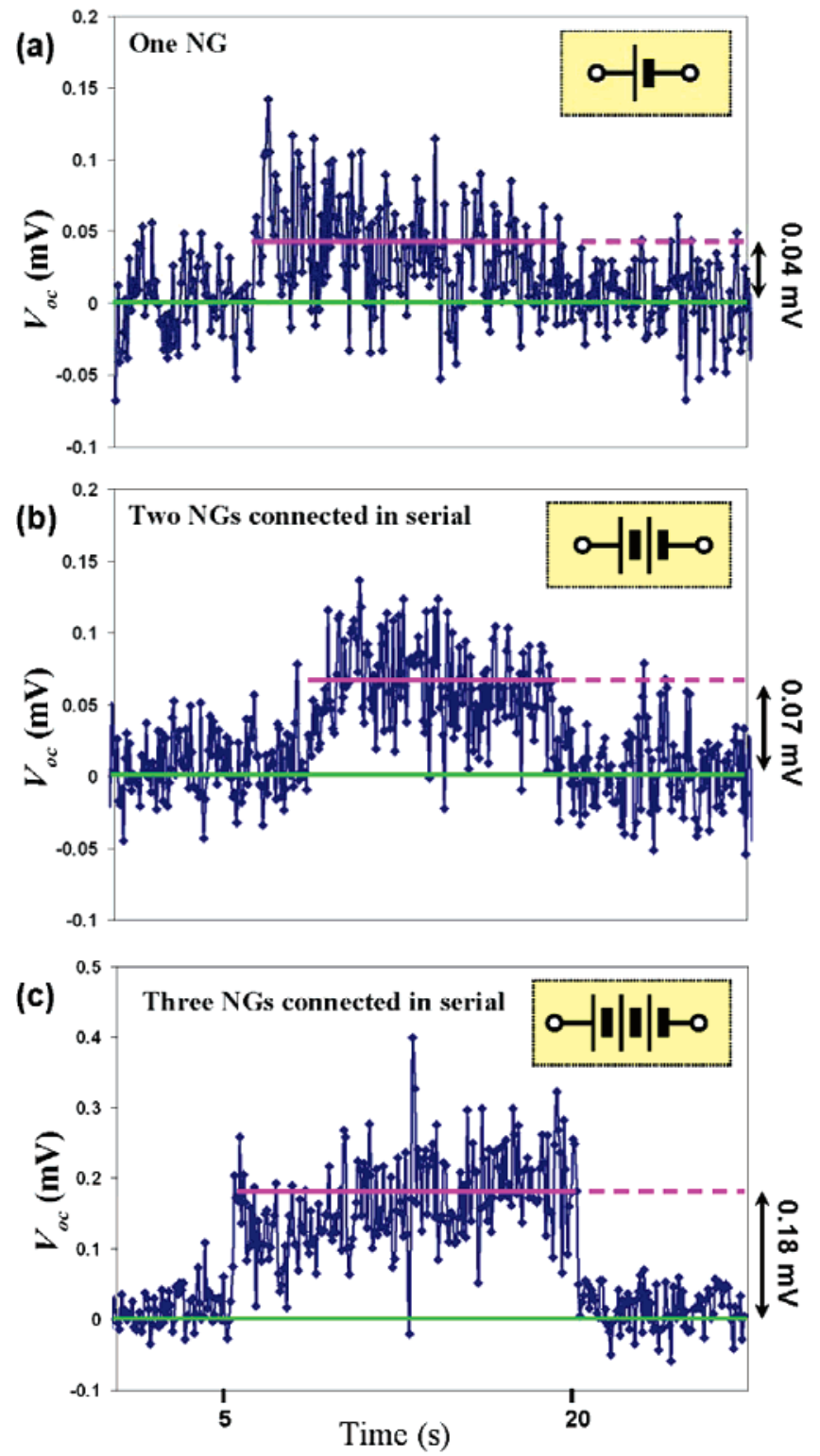

Figure 3. Open circuit voltage measured from an integrated NGs system. $(\mathrm{a}-\mathrm{c})$ Voltage signal measured from one, two, and three serial connected NGs. The connection configurations are schematically shown in the insets.

because the current relies on the number of NWs involved in power generating and the degree of bending of the NWs. It appears that the strong ultrasonic wave was effective for stimulating the output of the NG. The $35 \mathrm{nA}$ achieved here for a nanogenerator with an effective area of $2 \mathrm{~mm}^{2}$ is more than 30 times higher than that obtained in our previous report. ${ }^{9}$

The NG has a long lifetime. We have kept the ultrasonic wave on for $4 \mathrm{~h}$ without interruption, the NG was sustaining active for generating electricity without stop. We expect the lifetime of the NG is much longer than the time we have tested.

In summary, we have demonstrated a prototype NG that can effectively generate electricity inside biofluid when stimulated by ultrasonic waves. The open circuit voltage and short circuit current were carefully measured to characterize (a)
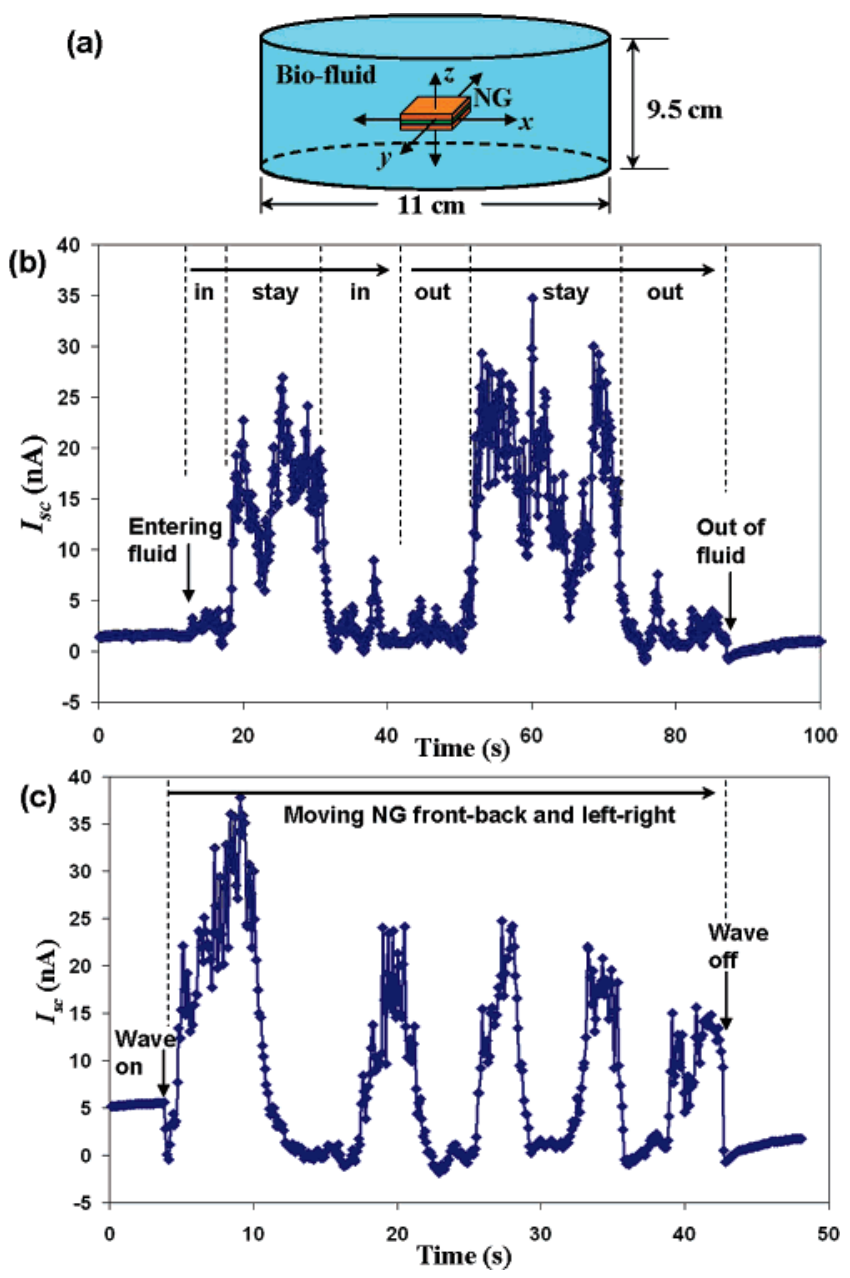

Figure 4. Enhanced output power by seeking the strong local intensity of the ultrasonic waves. (a) Schematic of the NG's position and moving directions inside biofluid. (b) Short circuit current signal measured during the movement of NG along the $z$ direction (from water surface to the bottom and then back to surface). (c) Short circuit current signal measured during the movement of NG along the $x$ and $y$ directions while the $z$ position was kept $3.3 \mathrm{~cm}$ below the water surface.

the NG's performance. The potential of increasing output current and voltage was illustrated by connecting multiple NGs in parallel and serial, respectively, unambiguously demonstrating the possibility of raising output power by three-dimensional integration and architecture. The effect of the local ultrasonic wave's intensity on the output was also studied. The output current was increased by 20-30 times and reached as high as $35 \mathrm{nA}$ when a $2 \mathrm{~mm}^{2} \mathrm{NG}$ was placed at the region where the ultrasonic waves were focused. This model successfully showed the solid feasibility of NG operation inside biofluid. It sets a solid foundation for selfpowering implantable and wireless biodevices and systems.

Acknowledgment. Thanks to the support of Georgia Research Alliance and Emory Georgia Tech CCNE (NIH).

\section{References}

(1) Patolsky, F.; Timko, B. P.; Zheng, G.; Lieber, C. M. MRS Bull. 2007, $32,142-149$.

(2) Patolsky, F.; Timko, B. P.; Yu, G.; Fang, Y.; Greytak, A. B.; Zheng, G.; Lieber, C. M. Science 2006, 313, 1100-1104. 
(3) Pauzauskie, P.; Yang, P. Mater. Today 2006, 9, 36.

(4) Paradiso, J. A.; Starner, T. Pervasive Computing 2005, 05, 18-27.

(5) Sales, B. C.; Mandrus, D.; Williams, R. K. Science 1996, 272, 13251328.

(6) Roundy, S.; Leland, E. S.; Baker, J.; Carleton, E.; Beilly, E.; Lai, E.; Otis, B.; Rabaey, J. M.; Wright, P. K.; Sundararajan, V. Pervasive Computing 2005, 05, 28-36.

(7) Bond, D. R.; Holmes, D. E.; Tender, L. M.; Lovley, D. R. Science 2002, 295, 483-485.
(8) Huynh, W. U.; Dittmer, J. J.; Alivisatos, A. P. Science 2002, 295, $2425-2427$

(9) Wang, X. D.; Song, J. H.; Liu, J.; Wang, Z. L. Science 2007, 316, $102-105$.

(10) Wang, Z. L.; Song, J. H. Science 2006, 312, 242-246.

(11) Song, J. H.; Zhou, J.; Wang, Z. L. Nano Lett. 2006, 6, 16561662.

NL0712567 